\title{
LETTER OPEN \\ Structural basis for the different states of the spike protein of SARS-CoV-2 in complex with ACE2
}

\author{
Cell Research (2021) 31:717-719; https://doi.org/10.1038/s41422-021-00490-0
}

Dear Editor,

Severe acute respiratory syndrome coronavirus 2 (SARS-CoV-2) has become a severe threat to global health. ${ }^{1}$ The spike $(S)$ protein on the surface of SARS-CoV-2 exploits angiotensin-converting enzyme 2 (ACE2) as cellular receptor and mediates the fusion of the viral and the host cell membrane during infection. ${ }^{1,2}$ The activation of the $S$ protein requires the receptor-binding domain (RBD) binding to the peptidase domain (PD) of ACE2 and the cleavage by host proteases into the $\mathrm{N}$-terminal $\mathrm{S} 1$ subunit and the C-terminal S2 subunit. ${ }^{2-4}$ An additional furin cleavage site "RRAR" present in the S1/S2 cleavage region of SARS-CoV-2, which is absent in SARS-CoV that shares about $80 \%$ sequence identity with SARS-CoV-2 and caused an epidemic in 2002-2003, might help accelerate the activation of the $S$ protein. $S 1$ consists of the $\mathrm{N}$-terminal domain (NTD), the RBD, subdomain 1 and 2 (SD1 and SD2). The RBD adopts two distinct conformations: "up" and "down". Only the "up" RBD exposes the receptor-binding site. S2, containing the hydrophobic fusion peptide, is responsible for the viral and cell membrane fusion. The molecular mechanism underlying the activation of the $\mathrm{S}$ protein of SARS-CoV-2 represents a critical clue for therapeutic development. Here we report the cryo-electron microscopy (cryo-EM) structures of trimeric extracellular domain of the $S$ protein (hereinafter referred to as S) at multiple states and conformations, including the all RBD-locked, activated, S1/S2 trypsin-cleaved, PD of ACE2-bound, and the full-length ACE2-bound states. PD binds to the trimeric $S$ protein with consistent interface, inducing conformational change at adjacent region. The trypsin-digested $S$ protein tends to accommodate more PD molecules. Furthermore, the structure of the $\mathrm{S}-\mathrm{ACE} 2-\mathrm{B}^{0} \mathrm{AT} 1$ ternary complex indicates that one ACE2 dimer binds two trimeric $S$ proteins simultaneously. These results provide clues for the mechanism of the activation of the $S$ protein of SARS-CoV-2.

We first solved the structure of the $S$ protein alone that was purified in different batches and exhibited two conformations: the locked conformation and the active conformation, with overall resolutions of $2.7 \AA$ and $3.3 \AA$, respectively. The locked conformation is similar to the previously reported structures, ${ }^{5-8}$ in which all RBD domains are in "down" position and compacted tightly, whereas in the active conformation, one of the RBD domains is in "up" position, which resembles the earliest reported structures $^{9,10}$ (Fig. 1a; Supplementary information, Figs. S1-S6 and Table S1). We did not observe the previously reported closed conformation, ${ }^{9}$ in which all RBD domains stay in "down" position but pack looser than that in the locked conformation. Transformation from the locked to the active state implies a tendency for S1 to become loose (Supplementary information, Video S1). The contact area between RBD and NTD of the S protein is reduced from the "down" RBD in locked conformation (locked-down) to the "down" RBD in active conformation (active-down) and to the "up" RBD in the active conformation (active-up) (Fig. 1b). The $S$ protein undergoes a clockwise twist from the locked conformation to the active conformation when looked from the $S$ protein to the viral membrane, which loosens the NTD and SD1 domain by increasing the distance between the individual domain and the central axis (Fig. 1c). For clarity, the clockwise direction and position are defined according to the same view.

To investigate the activation mechanism of the $S$ protein of SARS-CoV-2, we determined the structure of the $S$ protein incubated with PD of ACE2 in three different conformations with overall resolutions of 3.0-3.5 $\AA$ (Fig. 1d; Supplementary information, Figs. S1-S5 and Table S1), which account for $7.9 \%, 24.4 \%$ and $67.7 \%$ of particles, respectively. In conformation 1 , there is one "up" RBD, two "down" RBDs and no PD bound. In conformation 2, one RBD is in "up" conformation and bound by a PD molecule, whereas the other two RBDs are in "down" conformation. The conformation 2 resembles the previously reported S-PD complex. ${ }^{7}$ The conformation 3 is similar to conformation 2, except that the RBD clockwise near the PD-bound RBD is in "up" conformation and no PD molecule binds to it (Fig. 1d; Supplementary information, Video S2).

To investigate the effect of proteolytic processing on the activation of the S protein of SARS-CoV-2, we digested the purified $S$ protein by trypsin, which is referred to as $S(p)$ hereafter for clarity. We solved the structures of the $S(p)$ protein in complex with PD of ACE2 (Fig. 1e; Supplementary information, Video S3) with overall resolutions of 2.9-3.6 $\AA$, which show different PD binding results from the uncleaved S-PD complex. Three conformations of the S(p)-PD complex were observed. In conformation 1 that accounts for $9.5 \%$ particles, the $S(p)$ trimer is bound by only one PD molecule with two RBDs in "down" state. In conformations 2 and 3 that respectively account for $84.5 \%$ and $6 \%$ particles, the $S(p)$ trimer is bound by two PD molecules with the remaining RBD in "down" and "up" state, respectively. The binding of the second PD to the RBD of $S(p)$ in conformation 2 induces shift of both the NTD in the same protomer and the anticlockwise NTD compared with conformation 1 (Fig. 1f; Supplementary information, Fig. S7). Moreover, we found that the released S1 of S (p) could also bind to PD (Supplementary information, Figs. S3b, S8), which is consistent with the previous study. ${ }^{11}$ Based on these discoveries, we propose that the cleavage at S1/S2 site might affect the architectural rigidity of the $\mathrm{S}$ protein, making RBD more accessible to ACE2.

Additionally, we solved the structures of the $S(p), S(D 614 G)$, and the trypsin-digested $S(D 614 G)$ (hereinafter referred to as $S(p$, D614G)) proteins with overall resolutions of $3.2 \AA, 3.1 \AA$ and $3.3 \AA$, respectively, which all exhibit one RBD "up" conformation and show no obvious difference (Supplementary information, Fig. S9a). The result of the $S(p)$ protein is consistent with the previous study, which suggests that cleavage at the S1/S2 site facilitates the open conformation of RBD. ${ }^{4}$ The SARS-CoV-2 (D614G) mutant is associated with increased viral load in COVID-19 patients. ${ }^{12}$ To our surprise, $S(p, D 614 G)$ shows more digested bands, which means that the D614G mutation might increase the flexibility of the $S$ protein (Supplementary information, Fig. S9b). It is also reported that the D614G mutation can change the conformation 
a
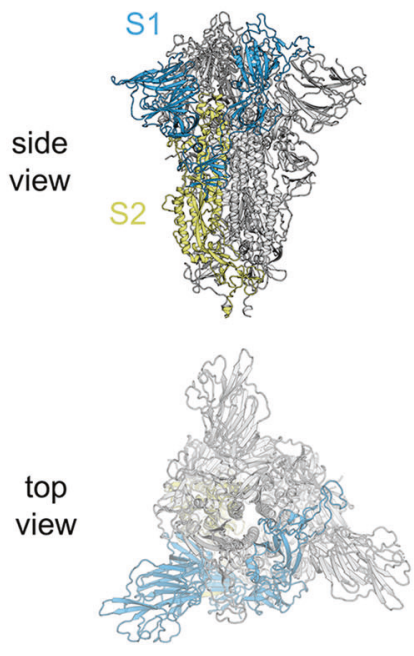

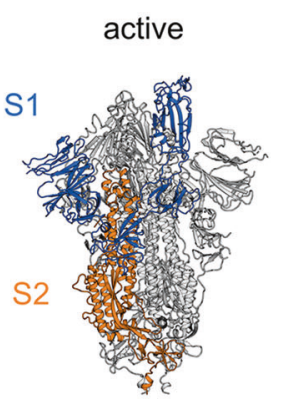

b
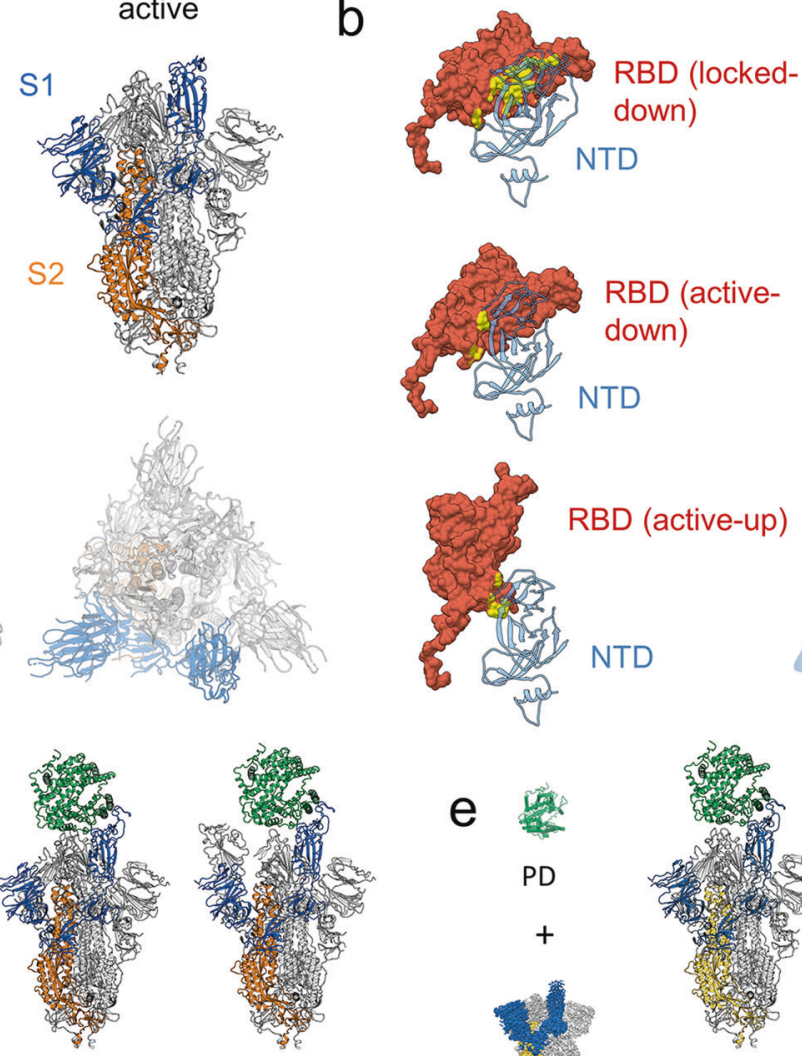

C3

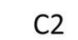

$7.9 \%$

$S$ protein

1 "up" RBD

no PD bound

$24.4 \%$

1 “up" RBD

1 PD bound

2 “up” RBD

1 PD bound
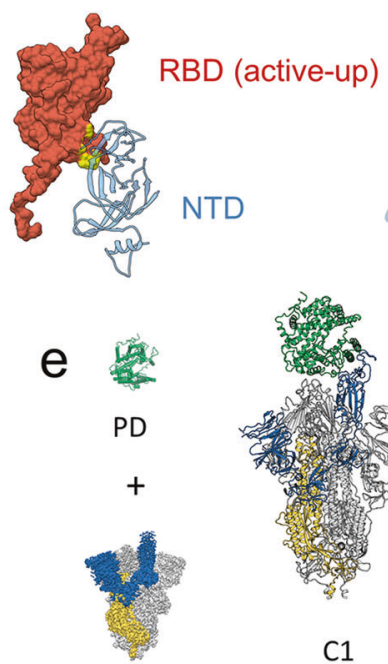

$S(p)$ protein

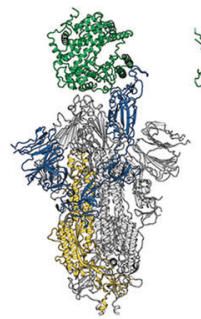

C1

$9.5 \%$

1 "up" RBD

1 PD bound

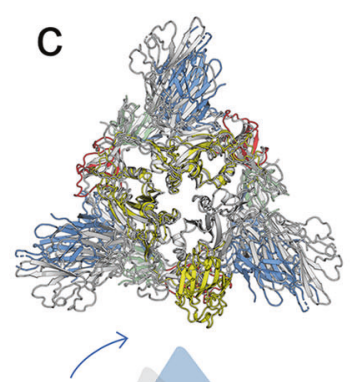

NTD
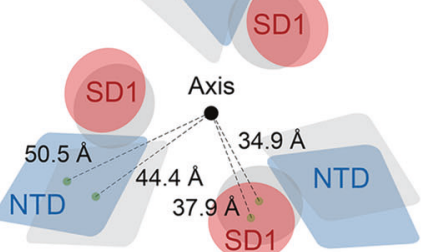
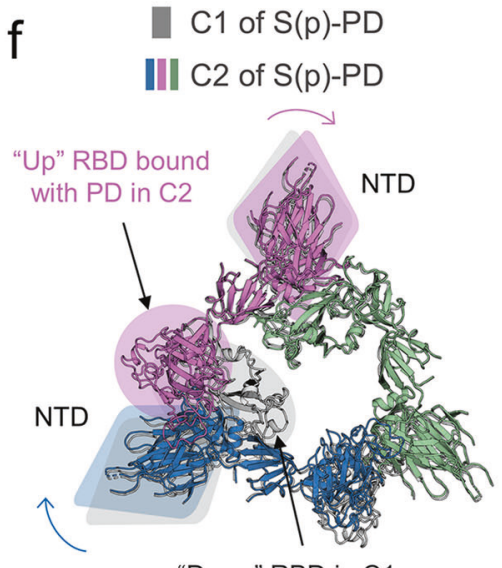

"Down" RBD in C1

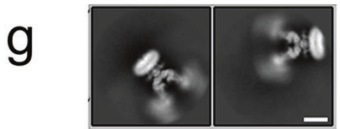

h

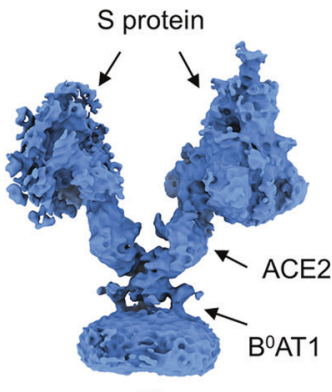

C1
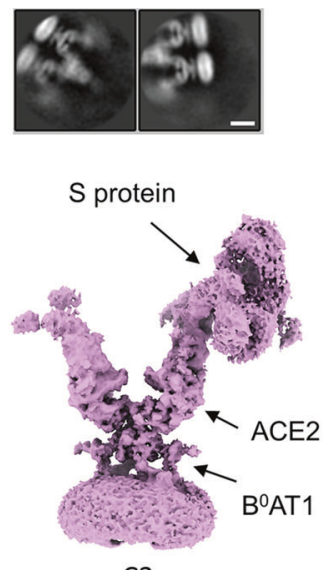

C2

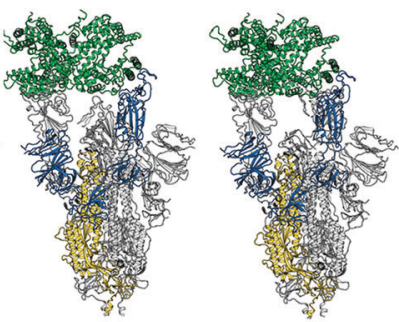

C3

$6 \%$

3 "up" RBD

2 PD bound

Fig. 1 Characterization of the interactions of the $S$ protein with ACE2. a Structures of the S protein. Left panel, locked conformation; right panel, active conformation with one RBD domain in the "up" position. b Decrease of contact area between RBD and NTD from the "down" RBD in the locked conformation (locked-down, upper panel), to the "down" RBD in the active conformation (active-down, middle panel), and to the "up" RBD in the active conformation (active-up, lower panel). c Structural comparison reveals a clockwise twist from the locked to the active conformation (upper panel), which loosens the contact between NTD and SD1 domain and increases the distance of these domains to the central axis. A schematic diagram is shown in the lower panel. Locked conformation is shown in gray. Active conformation is colored by domain, in which NTD is blue, RBD is yellow, SD1 is red and SD2 is green. d, e Different conformations of the S protein (d) and the S(p) protein (e) incubated with PD are shown, respectively. C1, C2 and C3 refer to conformation 1, 2 and 3, respectively. $\mathbf{f}$ Comparison between C1 and C2 of the S(p)-PD complex. Only RBD and NTD are shown for clarity. The binding of the second PD to RBD in C2 causes slight shift of NTDs both in the same protomer (violet) and in the anticlockwise protomer (blue). All protomers in C1 are colored in gray, whereas three protomers in C2 are colored in blue, violet and green, respectively. Circle and ellipse, RBD; diamond, NTD. g 2D class averages of the S-ACE2-B ${ }^{\circ}$ AT1 ternary complex are shown. Scale bar, $10 \mathrm{~nm}$. h Cryo-EM maps of conformation 1 (C1) and 2 (C2) of the S-ACE2-B ${ }^{\circ}$ AT1 ternary complex. i Docking model of conformation 1 of the S-ACE2-B AT1 ternary complex. 
of the $S$ protein to accommodate more PDs of ACE2. ${ }^{13}$ Taken together, the D614G mutation might increase the flexibility of the $S$ protein and make it easier to be digested by host protease, which might contribute to the increased infectivity.

Since PD of ACE2 exists as a monomer, whereas the full-length ACE2 presents as a dimer, a critical question is that how the fulllength ACE2 binds to the $S$ protein. In order to investigate the interactions of the S protein with ACE2 in a condition more closely resembling the physiological condition, we tried to solve the structure of the $S$ protein in complex with the full-length ACE2. We prepared and purified the S-full-length ACE2-B $A T 1$ ternary complex, which showed comigrating bands in size exclusion chromatography (SEC) analysis (Supplementary information, Fig. S10a). 2D classification exhibited blurred density on the top of each PD of ACE2 dimer, which was attributed to the S protein, suggesting that one ACE2 dimer can be bound by two $S$ proteins simultaneously (Fig. 1g, left panel). After 3D classification and refinement, we obtained two conformations for the S-ACE2-B ${ }^{0} A T 1$ ternary complex with resolutions of $8.3 \AA$ and $4.4 \AA$, respectively (Fig. 1h; Supplementary information, Figs. S10c-f, S11-S14). The right PD of the ACE2 dimer in both conformations is clearly bound by one $S$ protein, whereas the left PD of the ACE2 dimer is bound by a much more blurred $S$ protein and by a much smaller density in conformation 1 and 2, respectively. The local resolutions for ACE2, the left $S$ protein and the right $S$ protein in conformation 1 were improved to $8.4 \AA, 10.4 \AA$ and $6.7 \AA$, respectively (Supplementary information, Figs. S11, S13, S14a, C), which allowed a docking model for the S-ACE2-B ${ }^{0}$ AT1 ternary complex (Fig. 1i). This finding confirmed our previous hypothesis that one dimeric ACE2 might bind to two trimeric S proteins simultaneously (Fig. 1i; Supplementary information, Fig. S14a, c). ${ }^{3}$ Besides, the local resolutions for ACE2 and the right $S$ protein in conformation 2 were improved to $4.4 \AA$ and $4.3 \AA$, respectively (Supplementary information, Figs. S12$\mathrm{S} 14)$. As we were unable to determine the left density bound to ACE2 in conformation 2, we did not generate a model for this conformation.

The two PD domains in a dimeric full-length ACE2 cannot bind to the same trimeric $S$ protein because of the steric constriction, unless the RBD undergoes dramatic rotation as suggested by a previous study. ${ }^{14}$ It is not excluded that another ACE2 molecule can bind to the remaining RBD of the $S$ protein from another direction. Interestingly, we found additional 2D averages, in which multiple ACE2 dimers form a complex with different RBDs of one $S$ protein (Fig. 1g, right panel), consistent with a model proposed in the previous study. ${ }^{14}$ Additionally, the flexibility of the $S$ protein on the top of ACE2 and the previously reported transition of ACE2 between the open and closed conformations might be important factors for the activation of the $\mathrm{S}$ protein during infection. ${ }^{3}$

\section{DATA AVAILABILITY}

Atomic coordinates and cryo-EM density maps of conformation 1 of the S-ACE2-B ${ }^{0}$ AT1 ternary complex (PDB: 7DWX, whole map: EMD-30888, map focused on ACE2: EMD-30967, map focused on the left S protein: EMD-30966, map focused on the right S protein: EMD-30965) and conformation 2 of the S-ACE2- $\mathrm{B}^{0} \mathrm{AT} 1$ ternary complex (whole map: EMD-30968, map focused on ACE2: EMD-30970, map focused on the right S protein: EMD-30969) have been deposited to the Protein Data Bank (http://www.rcsb.org) and the Electron Microscopy Data Bank (https://www.ebi.ac.uk/ pdbe/emdb/), respectively. The other PDB and EMDB IDs can be found in Supplementary information, Table S1.

\section{ACKNOWLEDGEMENTS}

We thank the cryo-EM facility, the high-performance computing center and the mass spectrometry \& metabolomics core facility of Westlake University for providing supports. This work was funded by the National Natural Science Foundation of China
(32022037, 31971123, 81920108015, 31930059, 31800139), the SARS-CoV-2 emergency project of the Science and Technology Department of Zhejiang Province (2020C03129), the Key R\&D Program of Zhejiang Province (2020C04001), the China Postdoctoral Science Foundation (2020M681937 to R.Y.), the National Postdoctoral Program for Innovative Talents of China (BX20200304 to R.Y.), the Leading Innovative and Entrepreneur Team Introduction Program of Hangzhou, Westlake Education Foundation and Tencent Foundation.

\section{AUTHOR CONTRIBUTIONS}

Q.Z. and R.Y. conceived the project. Q.Z and R.Y. designed the experiments. All authors conducted experiments and contributed to data analysis. Q.Z. and R.Y. wrote the manuscript.

\section{ADDITIONAL INFORMATION}

Supplementary information The online version contains supplementary material available at https://doi.org/10.1038/s41422-021-00490-0.

Competing interests: The authors declare no competing interests.

Renhong Yan ${ }^{1,2}$, Yuanyuan Zhang ${ }^{1,2}$, Yaning $\mathrm{Li}^{3}$, Fangfei Ye ${ }^{1,2}$, Yingying Guo ${ }^{1,2}$, Lu Xia ${ }^{1,2}$, Xinyue Zhong ${ }^{1,2}$, Ximin Chi ${ }^{1,2}$ and Qiang Zhou ${ }^{1,2}$

${ }^{1}$ Center for Infectious Disease Research, Westlake Laboratory of Life Sciences and Biomedicine, Key Laboratory of Structural Biology of Zhejiang Province, School of Life Sciences, Westlake University, Hangzhou, Zhejiang 310024, China; ${ }^{2}$ Institute of Biology, Westlake Institute for Advanced Study, Hangzhou, Zhejiang 310024, China and

${ }^{3}$ Beijing Advanced Innovation Center for Structural Biology, Tsinghua-Peking Joint Center for Life Sciences, School of Life Sciences, Tsinghua University, Beijing 100084, China These authors contributed equally: Renhong Yan, Yuanyuan Zhang, Yaning $\mathrm{Li}$

Correspondence: Renhong Yan (yanrenhong@westlake.edu.cn) or Qiang Zhou (zhouqiang@westlake.edu.cn)

\section{REFERENCES}

1. Wu, F. et al. Nature 579, 265-269 (2020).

2. Hoffmann, M. et al. Cell 181, 271-280 (2020).

3. Yan, R. et al. Science 367, 1444-1448 (2020).

4. Wrobel, A. G. et al. Nat. Struct. Mol. Biol. 27, 763-767 (2020).

5. Toelzer, C. et al. Science 370, 725-730 (2020).

6. Xiong, X. et al. Nat. Struct. Mol. Biol. 27, 934-941 (2020).

7. Xu, C. et al. Sci. Adv. 7, eabe5575 (2020).

8. Zhou, T. et al. bioRxiv https://doi.org/10.1101/2020.07.04.187989 (2020).

9. Walls, A. C. et al. Cell 181, 281-292 (2020)

10. Wrapp, D. et al. Science 367, 1260-1263 (2020).

11. Benton, D. J. et al. Nature 588, 327-330 (2020).

12. Korber, B. et al. Cell 182, 812-827 (2020).

13. Yurkovetskiy, L. et al. Cell 183, 739-751 (2020).

14. Barnes, C. O. et al. Cell 182, 828-842 (2020).

\begin{abstract}
(c) (i)
Open Access This article is licensed under a Creative Commons Attribution 4.0 International License, which permits use, sharing, adaptation, distribution and reproduction in any medium or format, as long as you give appropriate credit to the original author(s) and the source, provide a link to the Creative Commons license, and indicate if changes were made. The images or other third party material in this article are included in the article's Creative Commons license, unless indicated otherwise in a credit line to the material. If material is not included in the article's Creative Commons license and your intended use is not permitted by statutory regulation or exceeds the permitted use, you will need to obtain permission directly from the copyright holder. To view a copy of this license, visit http://creativecommons. org/licenses/by/4.0/.
\end{abstract}

(c) The Author(s) 2021 